\title{
Steuerprogression in Deutschland - Annahmen und Wirklichkeit
}

\author{
Günter Britschkat \\ Charles C. Roberts
}

\begin{abstract}
Die vermeintliche Steuerbürde in Deutschland ist zum politischen Dauerthema geworden. Entsprechend populär sind die vielfältigen Vorschläge zur Reform des Steuerrechts. Allen Varianten gemeinsam ist das Ziel, die Steuerprogression abzubauen. Dieser Ansatz fußt auf der Vorstellung, die effektive Steuerlast entspräche den in den Steuerbestimmungen vorgesehenen hohen Progressionswerten. Dass dies mitnichten der Fall ist, wird im Folgenden empirisch nachgewiesen.
\end{abstract}

\section{Einleitung}

Die Steuerbürde war ein politisches Thema bei der letzten Bundestagswahl. Niemand zahlt gerne Steuern, und so ist der Ruf nach einer Verringerung der Steuerlast für sich immer populär. Die damit zumindest implizit erhobene Forderung nach einer Verringerung staatlicher Transfers bzw. Dienstleistungen ist indessen weniger populär. Auch wird eher nicht thematisiert, dass die Vor- und Nachteile von Steuersenkungen und Dienstleistungskürzungen die verschiedenen sozialen Gruppen sehr unterschiedlich treffen. Im Licht der politischen Diskussion stand hingegen die Steuerprogression, d.h. die in Bezug auf das Einkommen anteilsmäßige Zunahme des Steuersatzes bei höheren Einkommen. Verbreitet wurde behauptet, sie sei „zu hoch“ und würde die Initiative und die Bereitschaft zur Übernahme von Eigenverantwortung lähmen. Auch war im Wahlkampf die flankierende These zu hören, dass ausgleichende Korrekturen in der Einkommensverteilung ausschließlich - oder zumindest in der Hauptsache - der Steuerprogression bei der Besteuerung des persönlichen Einkommens vorbehalten sein sollten; andere sozialpolitische Systeme, wie beispielsweise die Gesundheits- oder Altersversorgung, sollten umverteilungsneutral gestaltet werden.

Ein Urteil über die Gültigkeit beider Argumente setzt aber eine zutreffende Vorstellung über das Ausmaß der effektiven Steuerbelastung im Allgemeinen voraus. Die Einkommensentwicklung und die sich daraus ergebende Entwicklung der Lohnund Einkommensteuerschuld des Einzel- nen sind indessen so stark tabuisiert, dass Vorstellungen über die effektive generelle Steuerprogression (im Gegensatz zu der eigenen) sehr stark von pauschalen Feststellungen und allenfalls vom Blick auf die Steuertabellen geprägt sind. Deswegen wollen wir im Folgenden gerade wegen der politischen Zusammenhänge der Frage nachgehen, wie stark die effektive Progression bei der Besteuerung der persönlichen Einkommen deutscher Haushalte in Abhängigkeit von Haushaltsgröße, sozio-ökonomischer Stellung und Haushaltseinkommen tatsächlich ist.

\section{Datenbasis}

Als Datenbasis dienen die Ergebnisse der Einkommens- und Verbrauchsstichprobe (EVS) des Statistischen Bundesamts (Statistisches Bundesamt 1998b; 2003b) für die Stichjahre 1998 und 2003. Die Daten sind für das frühere Bundesgebiet (FBG) und die neuen Bundesländer einschließlich Ost-Berlin (NBL), jeweils untergliedert hinsichtlich des Haushaltstyps, der sozialen Stellung des Haupteinkommensbeziehers und der Haushaltsgröße, verfügbar. Dabei ist jede Unterteilung wiederum gestaffelt nach Einkommensgrößenklassen. Die höchste Einkommensklasse wird auf ein Nettoeinkommen von 35.000 DM (1998), bzw. $18.000 €$ (2003) begrenzt.

Beim Haushaltstyp wird zwischen den Alleinlebenden, den Alleinerziehenden und den (Ehe-)Paaren unterschieden. Die soziale Stellung gliedert sich in Selbstständige (Landwirte, Gewerbetreibende, freiberuflich Tätige), Arbeitnehmer (Beamte, Angestellte, Arbeiter) und Nichterwerbs- tätige (Pensionäre, Rentner, Privatiers). Bei der Haushaltsgröße erfolgen die Darstellungen vom Ein-Personenhaushalt bis zum Vier(und mehr)-Personenhaushalt.

Die Anzahl der Einkommensklassen wird hier jeweils zu acht Klassen zusammengefasst. Dabei entsprechen die für das Jahr 1998 ausgewiesenen DM-Beträge recht genau den Euro-Werten des Jahres 2003.

Ein wichtiger Teil der Analyse bestand darin, für ,ausgepunktete“ Datenfelder in der amtlichen Veröffentlichung verlässliche Schätzwerte zu ermitteln. Hierzu und für weitere Einzelprobleme waren auch die Ergebnisse des Mikrozensus (Statistisches Bundesamt 1998a; 2003a), der jährlich erhoben wird, hilfreich. Für einige spezielle Fragestellungen erhielten wir vom Statistischen Bundesamt Zusatzinformationen. Dies betraf das Problem der Steuerrückerstattungen und die Fragen nach der Höhe von Kirchensteuer und Solidaritätsbeitrag.

Wenn im Weiteren von der Einkommensteuer die Rede ist, so ist die anteilige Kirchensteuer und der zugehörige Solidaritätsbeitrag stets mit inbegriffen.

Günter Britschkat, Wirtschaftswissenschaft-
ler im Ruhestand. Langjährig wissenschaft-
licher Mitarbeiter des Ifo-Instituts für
Wirtschaftsforschung.
e-mail: g.britschkat@gmx.de
Charles C. Roberts, Wirtschaftswissenschaft-
ler im Ruhestand. Langiährige Erfahrung in
der empirischen Wirtschaftsforschung;
Mitarbeit in der deutschen technischen
Zusammenarbeit.
e-mail: CRoberts@t-online.de
ler im Ruhestand. Langiährig wissenschaftlicher Mitarbeiter des Ifo-Instituts für Wirtschaftsforschung. Charles C. Roberts, Wirtschaftswissenschafter im Ruhestand. Langiährige Erfahrung in Mitarbeit in der deutschen technischen e-mail: CRoberts@t-online.de 


\section{Berechnung der kalkulatorischen Steuern}

Ziel der Untersuchung ist es, für jede Einkommensklasse einer jeden Kategorie eine theoretische Einkommensteuer zu ermitteln, die dann dem empirisch ermittelten Wert der EVS gegenübergestellt wird. Von besonderem Interesse ist dabei der Vergleich des Progressionsverhaltens der theoretischen und der empirischen Einkommensteuerbeträge.

Der in der EVS dargestellte Durchschnittshaushalt ergibt sich aus einer Vielzahl von Haushalten mit unterschiedlichen Steuermerkmalen. Die Steuerklassen unterscheiden sich, die Steuerfreibeträge divergieren und das zu versteuernde Einkommen schwankt innerhalb der Klassengrenzen. Wie kann unter diesen Umständen in sinnvoller Weise ein kalkulatorischer Steuerbetrag für den einzelnen Durchschnittshaushalt ermittelt werden?

Wesentlich für alles Weitere ist die Annahme, dass alle Steuerschuldner so behandelt werden, als wären sie Arbeitnehmer mit genau definierten Steuerfreibeträgen. Als Freibeträge werden hier berücksichtigt:

- die Pauschale für Werbungskosten der Arbeitnehmer (\$ 9a Nr.1 EStG),

- die jeweilige Vorsorgepauschale $(\$ 10$ Abs. 3 EStG),

- der Haushaltsfreibetrag (betrifft nur die Steuerklasse II) ( $\$ 32$ Abs. 7 EStG),

- der Kinderfreibetrag und die Kinderbetreuungskosten $(\$ 32$ Abs. 6, X. Abschn. EStG).

Die Pauschale für Werbungskosten der Arbeitnehmer wird, auch wenn beide Ehepartner arbeiten, nur einmal berücksichtigt. Die in der EVS ausgewiesenen Einkommensteuerzahlungen werden mit den gesondert publizierten Steuerrückerstattungen saldiert. Der Saldo zeigt also die Einkommensteuerlast nach Steuerbescheid.

Für die zu berechnende theoretische Steuerschuld sind somit nur drei Steuerklassen, und zwar I, II und III, zu berücksichtigen:

- Steuerklasse I (Alleinlebende, unverheiratete Paare ohne Kind),

- Steuerklasse II (Alleinerziehende),
- Steuerklasse III (Verheiratete Paare, mit Kindern oder ohne).

Für die verschiedenen Haushaltstypen ist die Zuordnung der passenden Steuerklasse problemlos. Schwieriger wird es, für die Merkmalsausprägungen der „Sozialen Stellung" und der „Haushaltsgröße" die richtigen Gewichtungen der einzelnen Steuerklassen zu finden. Hier hilft der Mikrozensus mit seinen tiefgegliederten Kreuztabellen weiter. Beispielsweise findet man so für die Gruppe der „Angestellten“ die Anteile der „Alleinlebenden“, der „Alleinerziehenden“ und der „Verheirateten“. Diese werden zur Gewichtung der einzelnen Steuerklassen verwendet.

Die Steuer für die einzelnen Einkommensklassen wird jeweils für das angegebene Durchschnittseinkommen berechnet. Dabei bleibt zunächst unberücksichtigt, dass die tatsächlichen Einkommen über die ganze Klassenbreite streuen können. Infolge der Progressionswirkung des Steuertarifs wird die theoretische Steuerbelastung somit unterschätzt. Wie Modellrechnungen zeigen, hat dieser "Splittingeffekt" eine Spannweite von 0 bis etwa $2 \%$ des geschätzten Steueraufkommens. Nur in der untersten Einkommensklasse erreicht dieser Wert bis zu $10 \%$, da hier die Grenze zwischen Besteuerung und Nichtbesteuerung liegt.

Es ist hervorzuheben, dass es sich hier um das Bruttoeinkommen des Haushalts, also das Einkommen (vor Steuern) aus unselbstständiger sowie aus selbstständiger Arbeit, handelt. Ferner geht es um Einkommen aus Vermögen (beispielsweise aus Vermietung und Verpachtung) sowie um öffentliche und nicht öffentliche Transferzahlungen. Der Gang der Berechnung führt vom Bruttoeinkommen der EVS zum einkommensteuerrechtlich relevanten Bruttoeinkommen, von hier über das zu versteuernde Einkommen zur Einkommensteuer. Das steuerrechtlich relevante Bruttoeinkommen ergibt sich als

Bruttoeinkommen

minus Transfereinkommen

plus Ertragsanteil der öffentlichen Renten plus Ertragsanteil der Betriebsrenten plus Pensionen.

Das Transfereinkommen beinhaltet auch alle Einkommen, die dem Progressionsvorbehalt unterliegen. Hierzu gehören beispielsweise neben vielen anderen Positio- nen das Arbeitslosengeld, das Krankengeld und das Kurzarbeitsgeld. Auch wenn diese Einkommen selber nicht direkt zum steuerrechtlich relevanten Einkommen gehören, so erhöhen sie doch in gewissem Maße die Steuerschuld. Dieser Effekt bleibt hier unberücksichtigt. Das theoretische Steueraufkommen ist also eher etwas größer als im Folgenden ausgewiesen.

Bei der Berechnung des steuerrechtlich relevanten Bruttoeinkommens werden die öffentlichen Renten und die Betriebsrenten mit ihrem Ertragsanteil berücksichtigt. Dieser wird einheitlich mit $30 \%$ angesetzt, was einem Renteneintrittsalter von 62 Jahren entspricht. Die Pensionen dagegen zählen voll zum steuerrechtlich relevanten Bruttoeinkommen.

Der Solidaritätszuschlag wird jeweils nach dem Solidaritätszuschlaggesetz berechnet. Für die Kirchensteuer fehlt eine solche einheitliche rechtliche Regelung. Sie fällt nur für die Mitglieder der Kirchen an. Da in dieser Hinsicht sich die Verhältnisse in den Alten und Neuen Bundesländern grundlegend unterscheiden, werden für beide Ländergruppen unterschiedliche $\mathrm{Zu}$ schlagssätze verwendet.

Die hier berechnete theoretische oder „kalkulatorische“ Steuerbelastung ist als Grundlage zur Veranschlagung der in Deutschland praktizierten Steuerhinterziehung nicht geeignet und die Autoren haben sie zu diesem Zweck auch nicht berechnet. Vielmehr soll sie ein Maßstab sein, mit dem die effektive Steuerprogression verglichen werden kann. Die kalkulatorische Steuerbelastung gibt in etwa die Steuerprogression wieder, so wie sie aus den Steuerformeln bzw. -tabellen hervorgeht, wenn man die Auswirkung der zahlreichen Steuerausnahmentatbestände auf die tatsächlich geleistete Einkommensteuer beiseite lässt. Sie spiegelt wohl in etwa wider, wie die Steuerprogression im politischen Diskurs häufig dargestellt wird und wie sie sich viele Wähler vorstellen.

\section{Entwicklung der Ein- kommensteuerprogression Ost und West zwischen 1998 und 2003}

Jeder Steuerzahler kennt seine Steuerlast, und jeder kann anhand der Steuertabellen bzw. -formeln ausrechnen, um wie viel sei- 


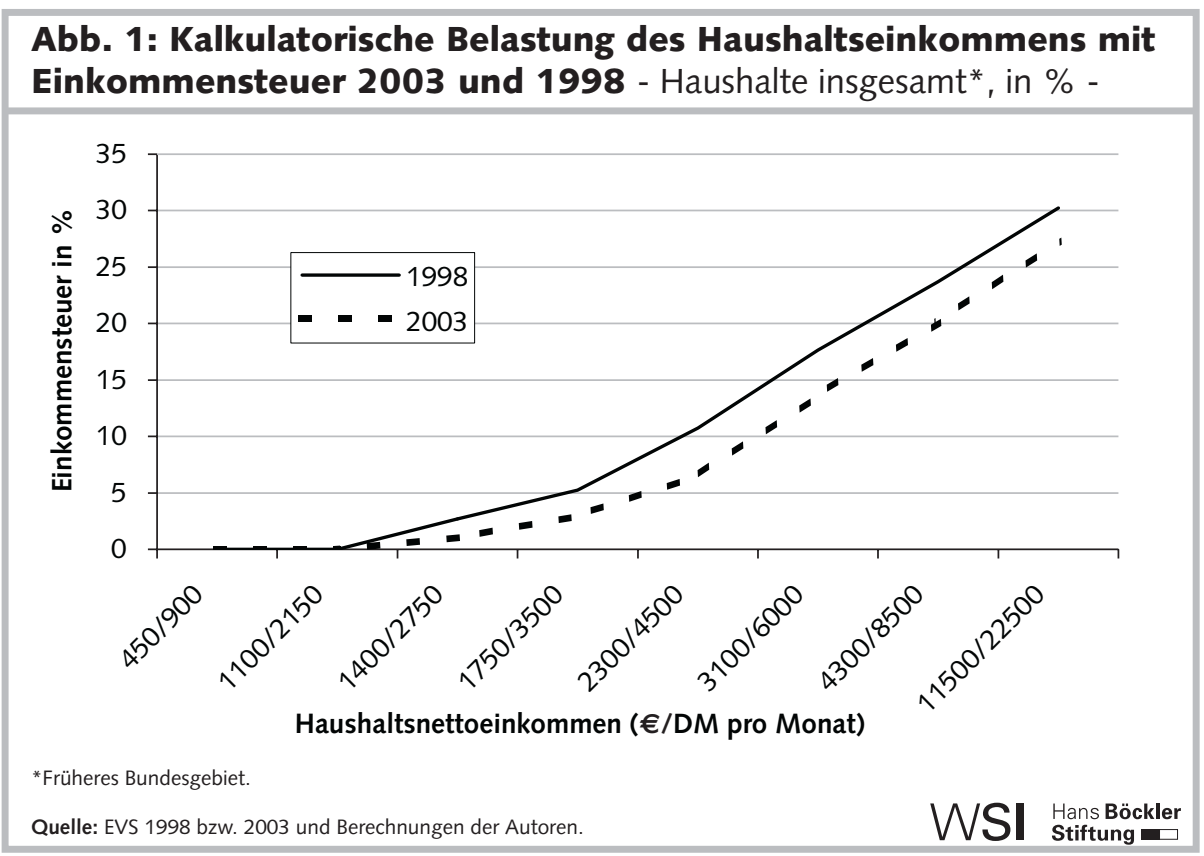

ne Steuerschuld zunehmen wird, wenn er oder sie $100 €$ mehr steuerpflichtiges Einkommen hätte. Solche Berechnungen werden gemeinhin unter einer ceteris paribusAnnahme aufgestellt: Nur das steuerpflichtige Bruttoeinkommen ändert sich; alle anderen steuerrelevanten Tatbestände werden als gleichbleibend angenommen. $A b$ bildung 1 gibt diesen Zusammenhang grafisch wieder. Aufgrund unserer Berechnungen der kalkulatorischen Einkommensteuerpflicht, die im Abschnitt 3 erläutert wurden, ergibt sich folgendes Bild, wobei die Belastung als Anteil am Haushaltsbruttoeinkommen dargestellt wird: ${ }^{1}$

Im Jahre 1998 fiel nach den Steuerformeln eine Steuerschuld erst ab einem Haushaltsbruttoeinkommen über 2.500 DM im Monat an. Zunächst war der Anstieg der Steuerbelastung relativ steil (auf ein Bruttoeinkommen von etwa 2.750 DM fiel eine Einkommensteuer von rund 2,5 \%; bei einem Bruttoeinkommen in Höhe von 3.500 DM waren nach der Tabelle bereits 2,7 \% des Haushaltseinkommens als Einkommensteuer zu entrichten. Schon am unteren Ende der Einkommenspyramide deuteten die damals gültigen Steuerformeln darauf hin, dass auf 100 DM zusätzliches Einkommen 22 DM mehr an Einkommensteuer zu zahlen waren. Aus Abbildung 1 geht ebenfalls hervor, dass die Steuerprogression weiter zunahm. Am oberen Ende der von der EVS erfassten Einkommensskala, welche freilich die wirklich hohen Einkommen nicht berücksichtigt, ist die Steuerpflicht anteilsmäßig deutlich
Haushaltsbruttoeinkommen von $5.400 €$ $1.090 €$ an Einkommensteuer zu entrichten, was auf eine Steuerlast von $20 \%$ hinausläuft. Bei einem Monatseinkommen von brutto $8.800 €$ fällt eine kalkulatorische Steuerschuld in Höhe von $2.400 €$ an, eine Steuerbürde von $27,3 \%$. Bei dieser Einkommenshöhe zieht der Steuerformel zufolge eine Bruttoeinkommensverbesserung um $100 €$ zusätzliche Einkommensteuerverpflichtungen in Höhe von $39 €$ nach sich.

Diese Zahlen beziehen sich auf Berechnungen einer theoretischen oder - wie wir es hier nennen - kalkulatorischen Steuerschuld, welche die zahlreichen Möglichkeiten, das steuerpflichtige Einkommen zu verringern, nicht berücksichtigen. Diese Möglichkeiten hängen zu einem erheblichen Teil mit Steuerbegünstigungen für besondere Einkommensverwendungen zusammen, die der Gesetzgeber als förderungswürdig ansieht (Beispiel: die inzwischen abgeschaffte Eigenheimzulage) oder mit besonderen Belastungen, die der Gesetzgeber mit Steuererleichterungen zumindest teilweise ausgleichen will (etwa die mittlerweile zurückgestutzte Pendlerpauschale). Der Fachbegriff für solche Steuerbegünstigungen ist ,Steuerausnahmetatbestand“; der Volksmund benutzt häufig den handlicheren Begriff „Steuergeschenk“. Bei der Diskussion um die Einkommensteuerprogression sollte es eigentlich auf die Progression der effektiven Besteuerung, also auf die tatsächlich geleisteten Steuern, ankommen. Die empirischen Angaben aus der EVS bieten eine Datenbasis zur Untersuchung dieses Themenkomplexes. $A b$ bildung 2 stellt kalkulatorische und effektive Steuerbelastung für Arbeitnehmerhaushalte (Arbeiter und Angestellte ohne Beamte) für die Jahre 1998 und 2003 gegenüber:

Abbildung 2 zeigt, dass im Jahre 1998 die effektive Steuerprogression vor allem schen Einkommens um. Ein Vergleich zwischen der kalkulatorischen Einkommensteuerpflicht aufgrund der reformierten Einkommensteuer für 2003 gegenüber 1998 zeigt, dass die neuen Bestimmungen im von der EVS erfassten Bereich die Progression kaum verschärft haben. Die Progression setzt erst bei einem höheren Einkommensniveau ein, ist zunächst etwas sanfter, aber bei Bruttohaushaltseinkommen über $2.000 €$ verläuft die neue Linie zwar unter der alten, jedoch grob parallel zu ihr. Im Jahre 2003 waren bei einem

\footnotetext{
Die 1998er Einkommensklassen sind in DM definiert; die für 2003 in Euro. Das Statistische Bundesamt hat zweckmäßigerweise für die zwei Stichjahre Einkommensklassen festgelegt, die sich grosso modo entsprechen; allerdings wird dabei die Geldentwertung zwischen 1998 und 2003 nicht berücksichtigt.

In der Grafik werden die Mittelpunkte der Einkommensklassen auf der Abszisse eingetragen. Um in der Systematik der EVS zu bleiben, werden auf der Abszisse die Haushaltsnettoeinkommensklassen dargestellt.
} 
Haushalte mit einem monatlichen Nettoeinkommen von weniger als $3.000 \mathrm{DM}$ betraf; höhere Einkommensklassen leisteten tatsächlich eine relativ niedrigere Einkommen- bzw. Lohnsteuer; erst bei Haushaltsbruttoeinkommen über etwa 6.000 DM im Monat fängt eine gewisse, allerdings recht sanfte Progression wieder an (durchgezogene Linie). Dennoch weisen die Angaben aus der 1998er EVS darauf hin, dass die Arbeitnehmerhaushalte in der höchsten Einkommensklasse (Haushaltsnettoeinkommen zwischen 10.000 und 35.000 DM) im Durchschnitt Einkommensteuer in Höhe von knapp 2.450 DM entrichteten. D.h., ihre Einkommen wurden anteilig effektiv geringfügig weniger belastet als die der Arbeitnehmerhaushalte in der Einkommensklasse 2.500 bis $3.000 \mathrm{DM}$ (13,8 gegenüber $14,7 \%$ ). Trotz der stark progressiven ,theoretischen" oder kalkulatorischen Steuerbelastung greift die effektive Progression vor allem in den unteren Einkommensklassen; bereits in der unteren Mitte der Einkommenspyramide ist bei Arbeitnehmern die effektive Belastung recht bescheiden. Ein zweiter Blick auf die Abbildung zeigt, dass im Progressionsbereich, d.h. Monatseinkommen bis zur Höhe von etwa 3.000 DM, die effektive Belastung, auch unter Berücksichtigung der von den Steuerzahlern erhaltenen Steuererstattungen, über der kalkulatorischen liegt. Beide Tatbestände verdienen eine genauere Betrachtung, aber zuerst sei die Frage erörtert, ob die 1999er Steuerreform hieran etwas Wesentliches verändert hat und ob die flache Belastungslinie auch andere sozioökonomische Gruppen betrifft.

Abbildung 2 zeigt die effektive Belastung im Jahre 2003 (graue durchgehende Linie). Man sieht (vgl. Lage und Verlauf der Linie), dass eine nennenswerte Verschärfung der effektiven Steuerprogression nicht $\mathrm{zu}$ erkennen ist; allenfalls in den niedrigsten Einkommensklassen (bis etwa $1.400 €$ Haushaltseinkommen) und dann wieder in den höchsten Einkommensklassen (oberhalb $5.000 €$ ) ist eine leicht steigende Tendenz zu sehen. Auch in dem Bereich der zunehmenden effektiven Progression ist die Grenzbelastung alles andere als horrend (auf $100 €$ zusätzliches Bruttoeinkommen erhöht sich im Durchschnitt die gesamte Steuerschuld um $23 €$ ). Die 2003er Ergebnisse zeigen auch eine, gemessen an der kalkulatorischen Belastung (gestrichelte Linie), im Verhältnis größer gewordene Überbezahlung in den unteren Einkom-

\section{Abb. 2: Belastung des Haushaltsbruttoeinkommens mit Einkommensteuer 1998 und 2003}

- Arbeitnehmerhaushalte insgesamt ${ }^{*}$, in \% -

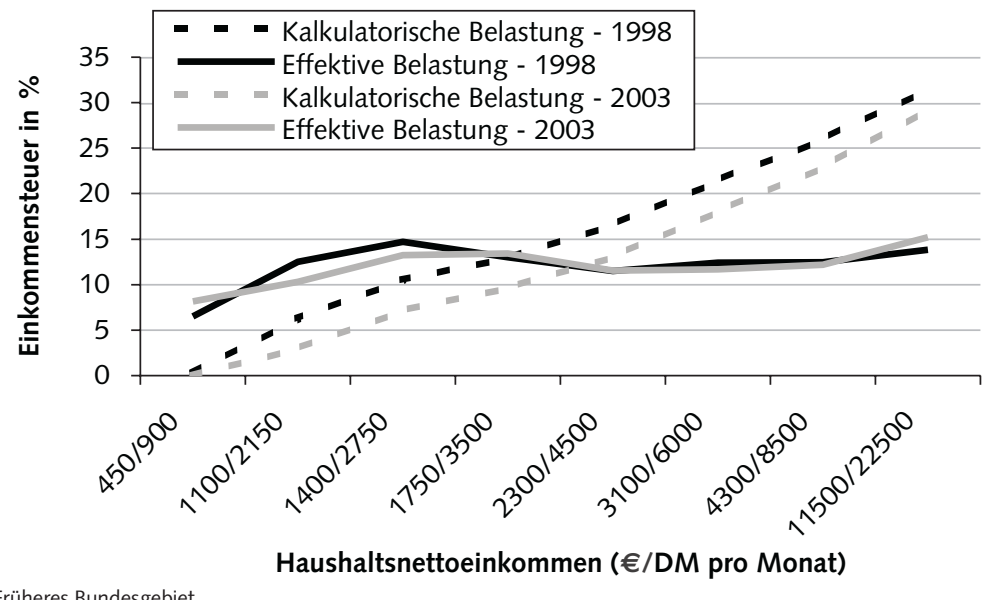

*Früheres Bundesgebiet.

Quelle: EVS 1998 bzw. 2003 und Berechnungen der Autoren.

WSI $\begin{gathered}\text { Hans Böckler } \\ \text { Stiftung }\end{gathered}$

mensklassen an. Bei einem durchschnittlichen Bruttohaushaltseinkommen in Höhe von $4.600 €$ monatlich gaben die Arbeitnehmerhaushalte im Durchschnitt 13,0 \% ihres Einkommens an den Staat als Einkommen- bzw. Lohnsteuer ab. Für alle 14,6 Mio. Arbeitnehmerhaushalte ergibt sich ein Steuerausfall, berechnet auf der Basis der kalkulatorischen Steuerbelastung, der auf 63,7 Mrd. € geschätzt wird; das sind $7,9 \%$ des Bruttoeinkommens dieser Haushaltsgruppe und $61 \%$ der effektiv geleisteten Einkommensteuer.

\subsection{BELASTUNG DES HAUSHALTS- EINKOMMENS DER SELBSTSTÄNDIGEN-HAUSHALTE}

Sucht man eine sozioökonomische Haushaltsgruppe, die eine sehr geringe effektive Progression und auch eine ausgeprägte Tendenz aufweist, bei ihren tatsächlich geleisteten Zahlungen die kalkulatorischen Steuerverpflichtungen noch deutlicher zu unterschreiten, so stößt man auf die Haushalte von Selbstständigen. Diese soziologisch sehr heterogene Gruppe umfasst Unternehmer im klassischen Sinne, aber auch Gewerbetreibende und Freiberufler, wie beispielsweise Ärzte bzw. Ärztinnen, RechtsanwältInnen, SchriftstellerInnen, und freischaffende KünstlerInnen. Entsprechend unterschiedlich ist die Zusammensetzung ihres Haushaltseinkommens: Während Unternehmer im klassischen Sinne, aber auch Gewerbetreibende, Gewinne aus den ihnen zugehörenden Unterneh- men (Personen- oder Kapitalgesellschaften) entnehmen, erhalten Freiberufler je nach Art der ausgeübten Tätigkeit Honorare, Vergütungen, Gagen etc. Die in der EVS ausgewiesenen Einkommensarten sind für diese sozio-ökonomische Haushaltsgruppe grundsätzlich nichts anders als für Arbeitnehmerhaushalte, doch die anteilige Zusammensetzung ihres Haushaltsbruttoeinkommens ist naturgemäß anders als die von Haushalten, deren Hauptverdiener Lohn- oder Gehaltsempfänger sind. Wir haben es hier mit dem Haushaltseinkommen und dessen Besteuerung im Zuge der Veranlagung zur Einkommensteuer zu tun. Die EVS bietet keine Datengrundlage, um bei den Selbstständigenhaushalten die Besteuerung des persönlichen Einkommens und die des unternehmerischen Gewinnes als Einheit zu betrachten. Für unsere Fragestellung das Ausmaß der effektiven Steuerprogression - ist dies jedoch belanglos, denn mit ihren einheitlichen Sätzen ist die Besteuerung der Unternehmensgewinne von Haus aus nicht progressiv gestaltet.

Für die Haushalte von Selbstständigen sah die Belastung des Haushaltsbruttoeinkommens mit Einkommensteuer im Jahre 2003 wie in Abbildung 3 dargestellt aus. Gegenüber 1998 hat es für diese sozioökonomische Gruppe keine ins Gewicht fallenden Verschiebungen gegeben. Da die Einkommensteuersätze für das Einkommen dieser Gruppe in keiner Weise von den Sätzen abweichen, die für andere Gruppen von Erwerbstätigen gelten, ist die extrem nied- 
rige und vergleichsweise flache Lage der Linie der effektiven Belastung vor allem darauf zurückzuführen, dass die Chancen, die effektiv entrichteten Steuern auf das persönliche Einkommen zu verringern, günstiger sind als beispielsweise in der Gruppe der Arbeitnehmer. Dies kann zum einen an der Vermögenslage von Selbstständigen, zum anderen an den Möglichkeiten der Steuerminderung liegen, die gewerbliche Aktivitäten bieten.

Tabelle 1 stellt einen Vergleich zwischen dem Steuergebaren der westdeutschen Selbstständigenhaushalte und dem der Arbeitnehmerhaushalte dar. Die Arbeitnehmerhaushalte, die vorab behandelt und kommentiert wurden, sind gewiss kein leuchtendes Beispiel für hohe Steuermoral, aber sie schneiden im Vergleich zu den Selbstständigen in Punkto Steuermoral glänzend $\mathrm{ab}$.

\subsection{UNTERSCHIED ZWISCHEN KALKULATORISCHER UND EFFEKTIVER BELASTUNG}

Was das Auseinanderklaffen von kalkulatorischer und effektiver Belastung in den höheren Einkommensklassen betrifft (ein Phänomen übrigens, das sich durch alle untersuchten sozioökonomischen Haushaltsgruppen zieht), so gibt es prinzipiell drei Gründe:

(1) Steuerprivilegierte Einkommensverwendungen;

(2) Steuerrelevante Beeinflussung der Art und Weise, wie Einkommen entsteht;

(3) Steuerhinterziehung.

$\mathrm{Zu}$ (3) ist festzustellen, dass Steuerhinterziehung, ob illegal oder staatlich toleriert, mit Sicherheit im Spiel ist, obgleich Haushalte, die an einer amtlichen Haushaltsbefragung über ihre Einkommensverhältnisse freiwillig teilnehmen, höchstwahrscheinlich nicht die Vorhut dieses neuen Volkssports bilden. Wie dem auch sei, die EVS bietet keine Datengrundlage zur quantitativen Erfassung der im Lande praktizierten Steuerhinterziehung.

Es gibt mehrere Möglichkeiten, die Art und Weise, wie Einkommen entsteht, mit Blick auf die Vermeidung von Steuern zu beeinflussen. Eine davon ist die informelle Wirtschaft, in der Einkommen entsteht, das von den Steuerbehörden nicht erfasst wird, sodass die Entrichtung von Steuern

\section{Abb. 3: Belastung des Haushaltsbruttoeinkommens mit}

Einkommensteuer 2003 - Haushalte von Selbstständigen*, in \% -

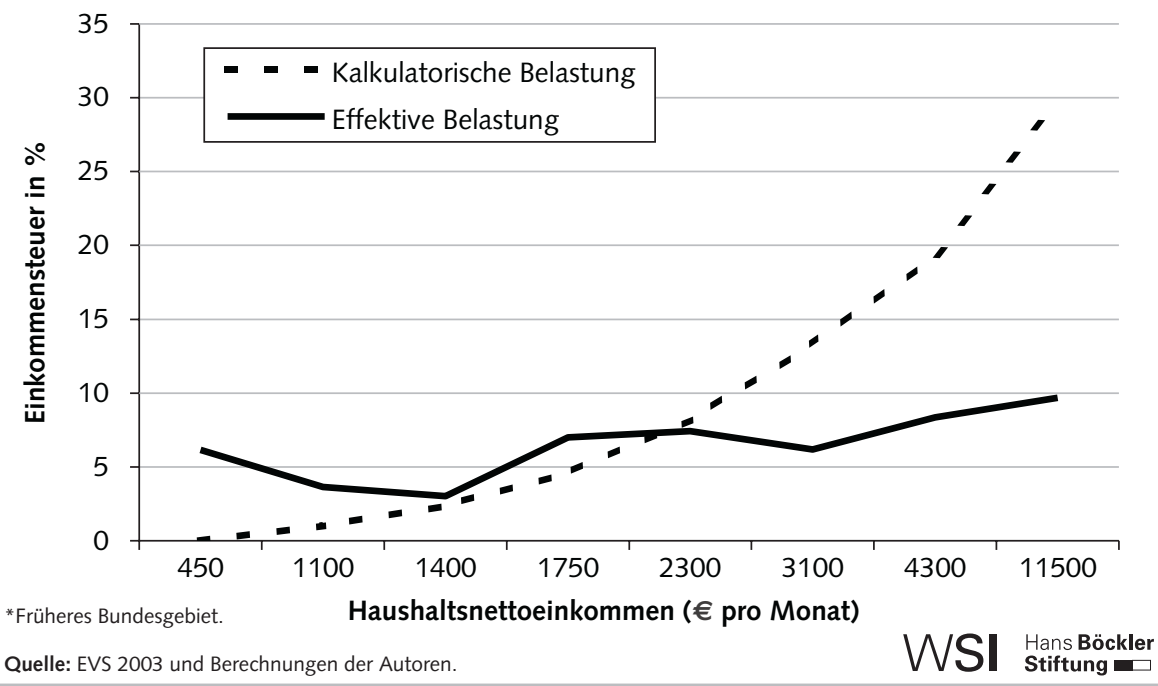

Tabelle 1: Steuerbelastung und Zahlungsmoral - Früheres Bundesgebiet 2003 -

\begin{tabular}{|c|c|c|c|}
\hline & $\begin{array}{l}\text { Selbstständigen- } \\
\text { haushalte }\end{array}$ & $\begin{array}{l}\text { Arbeitnehmer- } \\
\text { haushalte }\end{array}$ & $\begin{array}{l}\text { Arbeitnehmer- } \\
\text { haushalte in \% der } \\
\text { Selbstständigen- } \\
\text { haushalte }\end{array}$ \\
\hline Zahl der Haushalte (in 1.000) & 1.900 & 14.598 & - \\
\hline $\begin{array}{l}\text { Durchschnittliches monatliches } \\
\text { Bruttohaushaltseinkommen }\end{array}$ & $€ 5.443$ & $€ 4.600$ & $84,51 \%$ \\
\hline Kalkulatorische Steuerschuld & $€ 1.265$ & $€ 959$ & $75,81 \%$ \\
\hline $\begin{array}{l}\text { Effektiv geleistete Einkommensteuer } \\
\text { in \% des Bruttoeinkommens } \\
\text { in \% der kalk. Steuerschuld }\end{array}$ & $\begin{array}{r}\quad 474 \\
8,7 \% \\
37,5 \%\end{array}$ & $\begin{array}{l}€ 596 \\
13,0 \% \\
62,1 \%\end{array}$ & $\begin{array}{r}125,74 \% \\
- \\
-\end{array}$ \\
\hline
\end{tabular}

unterbleibt. Während der informelle Sektor auch dem „kleinen Mann“ unter Umständen eine Zuflucht vor Steuern bietet, gibt es für Menschen mit überdurchschnittlichem Geldvermögen noch die Möglichkeit, Geldvermögen außerhalb des Geltungsbereichs der deutschen Finanzämter anzulegen und somit die Erträge dieser Anlagen dem Zugriff des Fiskus zu entziehen. Die Länder an der Süd- und südwestlichen Grenze Deutschlands (Österreich, Liechtenstein, die Schweiz, Luxemburg) haben hier einen beachtlichen Finanzdienstleistungssektor aufgebaut, der mittlerweile einen wichtigen Platz in ihren Volkswirtschaften einnimmt. Das Ausmaß der Steuerausfälle infolge der hier gebotenen Möglichkeiten, sich der Steuerpflicht zu entziehen, ist naturgemäß schwer exakt zu beziffern, aber alles spricht dafür, dass es beachtlich ist. Der Verband der Bayerischen Genossenschaftsbanken ließ verlautbaren, dass im Jahre 2005 mindestens 434 Mio. $€$ aus ihren Instituten ins Ausland - in erster
Linie nach Österreich - verlagert würden. Der Verband führt die Geldvermögensflucht auf die Einführung des automatischen Kontenabrufs zugunsten der Finanzämter in Deutschland zurück. Unter dem Aspekt der Steuervermeidung ist vielleicht noch besorgniserregender, dass zumindest zwei Mitglieder des Bayerischen Verbands hierauf reagiert haben, indem sie Niederlassungen im Nachbarland einrichteten; weitere Bayerische Genossenschaftsbanken erwägen diesen Schritt; die Banken folgen dem Geld. Die dahinter steckende Überlegung ist die, dass Filialen deutscher Geldinstitute mit Sitz in Österreich das österreichische Bankgeheimnis - auch gegenüber deutschen Finanzämtern - in Anspruch nehmen können (Süddeutsche Zeitung 2006). Die 434 Mio. €, von denen der Verband berichtete, stammten wohlgemerkt nur aus Privatkonten in nur einem Bankbereich (Volks- und Raiffeisenbanken) in nur einem Bundesland (Bayern) während nur eines Jahres (2005). 
Dieses im Ausland angelegte Geldvermögen von deutschen Steuerbürgern entzieht sich dem Zugriff der deutschen Steuerbehörde nicht völlig, denn die ausländischen Banken in den europäischen Steueroasen sind seit Mitte 2005 angehalten, Abgaben auf anfallendes Einkommen aus diesen Guthaben einzubehalten und sie pauschal an die Länder abzuführen, in denen die Anleger ihren Hauptwohnsitz haben. Eine gewisse pauschale Besteuerung dieses Einkommens zugunsten des deutschen Staatsäckels findet also doch statt, doch die Regelung verhindert auch im $\mathrm{Zu}$ ge eines nachträglichen Ausgleiches eine progressiv gestaltete Besteuerung dieses hauptsächlich bei vermögenden Menschen anfallenden Einkommens. Denn die luxemburgischen, österreichischen etc. Geldinstitute schicken keine personenbezogenen Kontrollmitteilungen an die deutschen Finanzämter, die eine Besteuerung dieser Einkommensströme nach den Maßgaben des deutschen Steuerrechts ermöglichen würden. Die Banken in Österreich, der Schweiz und Luxemburg verteidigen verbissen ihr Bankgeheimnis und leisten selbst beim „Kavaliersdelikt“ Steuerhinterziehung keine Amtshilfe; erst beim viel gravierenderen Straftatbestand des Steuerbetrugs sind sie gegenüber deutschen Behörden auskunftspflichtig (Raupp 2006; Financial Times 2005). Angesichts dieser Sachlage scheint es gerechtfertigt, in Bezug auf die Besteuerung dieser Einkommen von staatlicherseits quasi geduldeter Steuervermeidung zu sprechen (um das hässliche Wort „Hinterziehung“ zu umgehen).

\subsection{FOLGEN STEUERPRIVILEGIERTER EINKOMMENSVERWENDUNGEN}

Bei den steuerprivilegierten Einkommensverwendungen ist es gänzlich unangebracht, von Steuerhinterziehungen zu sprechen, denn diese Möglichkeiten sind vom Gesetzgeber ausdrücklich gewünscht. Die Steuergesetze sehen zahlreiche Arten von Einkommensverwendungen vor, die einen Abzug von zu versteuerndem Einkommen begründen. Beispiele sind Aufwendungen für die Altersvorsorge, verschiedene Arten von Vermögensbildung: von der Investition in selbst bewohntes Wohneigentum bis hin zu Anlagen in Containerschiffe (seit einiger Zeit abgeschafft). Aber auch gewisse Konsumausgaben zählen hierzu, die einen Abzug begründen, weil sie eine Belastung darstellen, die der Gesetzgeber zumindest

\section{Abb. 4: Belastung des Haushaltsbruttoeinkommens mit Ein- kommensteuer 1998 und 2003 in Deutschland}

- Haushalte insgesamt, in \% -

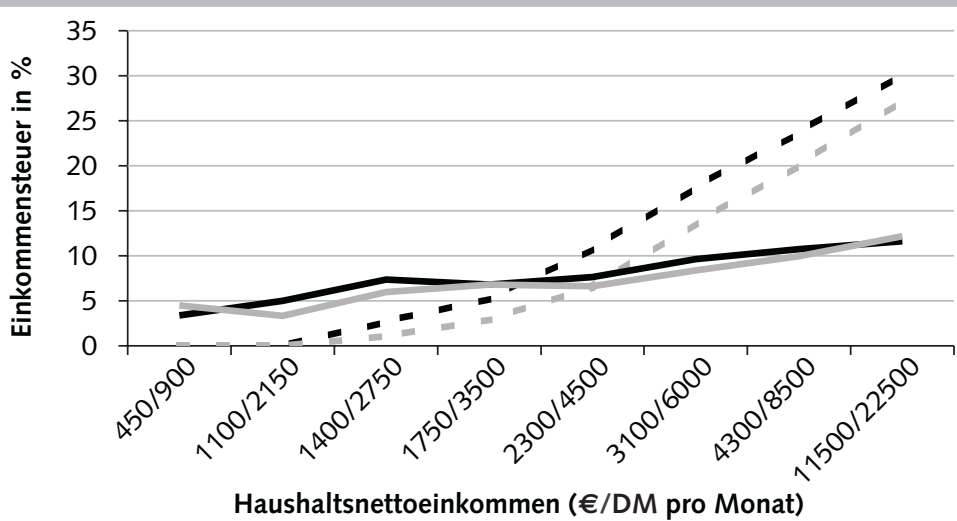

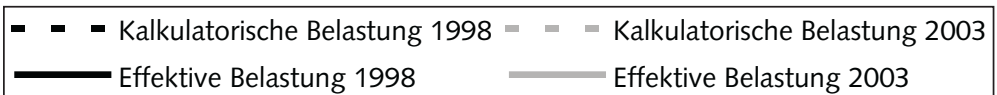

Quelle: EVS 1998 bzw. 2003 und Berechnungen der Autoren.

WSI $\begin{gathered}\text { Hans Böckler } \\ \text { Stiftung }\end{gathered}$

teilweise mit Steuererleichterungen kompensieren will (ein Beispiel wäre die mittlerweile zurückgestutzte Pendlerpauschale, die einen Abzug für Fahrtkosten zwischen Wohnort und Arbeitsstätte in gewissen Umfängen gestattet).

Es ist ein fester Grundsatz deutschen Steuerrechts, dass solche Steuerprivilegien als Abzug von zu versteuerndem Einkommen gewährt werden. Dies hat zur Folge, dass die eingetretene Entlastung vom Grenzsteuersatz abhängt, der beim besser Verdienenden höher liegt. So zieht bei einem Steuerzahler in der höchsten Steuerklasse eine steuerlich relevante Aufwendung in Höhe von $100 €$ eine Verringerung der Steuerschuld um $45 €$ nach sich; ein Durchschnittsverdienender, also einer, der den Eingangssatz entrichtet, erfährt bei gleicher Aufwendung eine Steuerminderung um lediglich $20 €$. Die Praxis, die Steuererleichterung über einen Abzug von zu versteuerndem Einkommen herbeizuführen, führt dazu, dass ein Einkommensbezieher, dessen steuerpflichtiges Einkommen unterhalb der Eingangsschwelle liegt, trotz steuerprivilegierter Ausgaben eben keine Steuererleichterung erfährt. Ein solcher Steuerbürger kommt naturgemäß nur selten in die Verlegenheit, beispielsweise selbstbewohntes Wohneigentum zu erwerben, aber es ist vorstellbar, dass er einen längeren Weg zur Arbeit haben könnte; die Pendlerpauschale brächte ihm indessen keinen Einkommensvorteil, oder anders gewendet: Ausgerechnet die ärmeren Einkommensbezieher, deren steuerpflichtige
Einkommen nach Berücksichtigung aller für ihn/sie geltenden Freibeträge und Ähnlichem unter der Eingangsschwelle liegen, erhalten über das Steuersystem keinen Ausgleich für Ausgaben, die bei höher Verdienenden eine Steuerentlastung und mithin eine Einkommensverbesserung mit sich bringen. ${ }^{2}$

Abbildung 4 fasst die Ergebnisse für ganz Deutschland zusammen. Was die kalkulatorische Belastung des Haushaltsbruttoeinkommens betrifft, so hat sich die Linie zwischen 1998 und 2003 deutlich verschoben. Die 2003er Bestimmungen sehen eine theoretische Progression vor, die zunächst deutlich sanfter ansteigt; der immer noch vorhandene steile Anstieg der kalkulatorischen Progression setzte im Jahre 2003 für die meisten Haushalte erst bei einem Haushaltsbruttoeinkommen von über $3000 €$ ein. Die aus der amtlichen Haushaltsumfrage gewonnenen Angaben zeigen indessen, dass die effektive Progression deutlich flacher als die kalkulatorische ausfällt; für

\footnotetext{
2 Plötzliche und unerwartete Einkommensverbesserungen können zunächst und vorübergehend zu einer Zunahme der Steuerbelastung führen, die der in den Steuertabellen vorgesehenen in etwa entspricht, wenn es dem Steuerzahler nicht gelingt, durch die Inanspruchnahme von Steuerausnahmetatbeständen die Zunahme seines steuerpflichtigen Einkommens zu begrenzen. Ein ganzer Berufsstand (Steuerberater, -anwälte) steht bereit, ihn bei seinen diesbezüglichen Bemühungen zu beraten. In der Regel wird es in relativ kurzer Zeit gelingen, den Anstieg der relativen Belastung aufzuhalten
} 
das von der EVS erfasste Einkommensspektrum lag sie 2003 in einer Bandbreite zwischen knapp 4 und $12 \%$ des Haushaltsbruttoeinkommens. Zunächst - d.h. beim Übergang von der niedrigsten zur nächst höheren Einkommensklasse - ist keine Progression erkennbar. Darüber hinaus hat steigendes Einkommen eine leicht steigende Steuerlast zur Folge, doch die Stichprobenergebnisse deuten darauf hin, dass selbst die Haushalte in der höchsten von der EVS erfassten Einkommensklasse trotz der für sie geltenden weit höheren kalkulatorischen Sätze eine Einkommensteuer effektiv zu entrichten haben, die im Durchschnitt bei unter $13 \%$ ihres Bruttohaushaltseinkommens liegt. Insgesamt betrachtet trat zwischen 1998 und 2003 eine äußerst geringe Steuerentlastung ein: Betrug doch die Steuerlast für alle von der EVS erfassten Haushalte 1998 9,3 \% ihres Bruttoeinkommens, so sank sie bis 2003 auf $9,1 \%$.

\section{Fazit}

Die weitverbreitete Auffassung, das persönliche Einkommen deutscher Haushalte würde einer ausgeprägt progressiven $\mathrm{Be}$ steuerung unterliegen, wird durch eine empirische Überprüfung anhand der Daten über die Einkommensverteilung und -verwendung aus der amtlichen Einkommensund Verbrauchsstichprobe des Statistischen Bundesamtes nicht bestätigt. Wohl ist eine an den tatsächlich entrichteten Steuern auf das Haushaltseinkommen gemessene effektive Progression noch vorhanden; sie ist aber eher als sanft zu bezeichnen. Auf jeden Fall liegt die effektive Steuerlast auf persönliches Einkommen weit unter der kalkulatorischen Last, die sich aus den Steuerformeln bzw. -tabellen ergibt. Die Gründe für das Auseinander- klaffen zwischen effektiver und kalkulatorischer Steuerlast sind in den zahlreichen Ausnahmetatbeständen, die das deutsche Steuerrecht bereithält, in den Möglichkeiten, Einkommen außerhalb des Zugriffs des deutschen Fiskus entstehen zu lassen, und schließlich in Steuerhinterziehung zu sehen. Die Aushöhlung der theoretisch vorgesehenen Steuerprogression zeigt, dass diese Möglichkeiten den Beziehern höherer Einkommen unverhältnismäßig zugute kommen. Die zur Zeit vorliegenden Vorschläge zur weiteren „Reform“ der Besteuerung des persönlichen Haushaltseinkommens gehen einhellig in Richtung weniger Progression in den Steuersätzen, einige Vorschläge sehen jedoch einen dem entgegengesetzten Abbau der die Progression aushöhlenden Steuerausnahmetatbestände vor.

\section{LITERATUR}

Bach, S. (2004): Reformkonzepte zur Einkommens- u. Ertragsbesteuerung: Erhebliche Aufkommens- u. Verteilungswirkungen, aber relative geringe Effekte auf das Arbeitsangebot, in: Wochenbericht des DIW, 15. April

Financial Times Deutschland (2005): Erzwungene Ehrlichkeit. Ab 1. Juli müssen Banken Kontrollmitteilungen versenden, doch die EU-Zinsrichtlinie hat viele Schlupflöcher, 28. Juni

Raupp, J. (2006): Tradition, Wissen und das Bankgeheimnis. Die Zinssteuer beeinflusst die Geldströme aus der EU kaum, die Depotwerte von ausländischen Kunden klettern auf neue Rekordhöhen, in: Süddeutsche Zeitung vom 29. März
Statistisches Bundesamt (1998a): Fachserie 1, Bevölkerung und Erwerbstätigkeit; Reihe 3, Haushalte und Familien Statistisches Bundesamt (1998b): Fachserie 15, Heft 4, Einkommensund Verbrauchsstichprobe

Statistisches Bundesamt (2003a): Fachserie 1, Bevölkerung und Erwerbstätigkeit; Reihe 3, Haushalte und Familien

Statistisches Bundesamt (2003b): Fachserie 15, Heft 4, Einkommensund Verbrauchsstichprobe

Süddeutsche Zeitung (2006): Bayern beklagen Kapitalflucht. Genossenschaftsbanken wollen verstärkt nach Österreich gehen, 10. März 